\title{
Association of Continuous Vertebral Bone Bridges and Bone Mineral Density with the Fracture Risk in Patients with Diffuse Idiopathic Skeletal Hyperostosis
}

\author{
Mitsuru Furukawa ${ }^{1}$, Kunimasa Okuyama $^{2}$, Ken Ninomiya ${ }^{2}$, Yoshiyuki Yato ${ }^{1}$, \\ Takeshi Miyamoto ${ }^{3}$, Masaya Nakamura ${ }^{4}$, Morio Matsumoto ${ }^{4}$ \\ ${ }^{1}$ Department of Orthopedic Surgery, Murayama Medical Center, Tokyo, Japan \\ ${ }^{2}$ Department of Orthopedic Surgery, Shizuoka City Shimizu Hospital, Shizuoka, Japan \\ ${ }^{3}$ Department of Orthopedic Surgery, Kumamoto University, Kumamoto, Japan \\ ${ }^{4}$ Department of Orthopedic Surgery, Keio University, Tokyo, Japan
}

\section{Study Design: Cross-sectional study.}

Purpose: To examine whether the number of continuous vertebral bone bridges and bone mineral density (BMD) influence the fracture risk in diffuse idiopathic skeletal hyperostosis (DISH) patients.

Overview of Literature: Bone bridges connecting through the intervertebral body in DISH create long lever arms that can increase the risk of fractures from minor trauma. DISH patients have a BMD that is higher than or comparable to those of age-matched healthy subjects.

Methods: We examined the computed tomography scans from the thoracic vertebra to the sacrum used to diagnose DISH in 140 patients ( 98 men and 42 women; average age, 78.6 years). We compared patients who did ( $n=52$ ) and did not have $(n=88)$ fractures at the continuous vertebral bodies fused by bone bridges. The relationship between the vertebral fractures and the maximum number of vertebrae that are bony cross-linked with contiguous adjacent vertebrae ( $\max V B$ ) from the thoracic vertebra to the sacrum or from the lumbar vertebra to the sacrum and proximal femur BMD were analyzed using a logistic regression model.

Results: We found that after adjusting for the confounding factors, higher max VB, both from the thoracic vertebrae to the sacrum and the lumbar vertebrae to the sacrum, was associated with a higher risk of vertebral fractures. This difference was statistically significant. The risk was higher when only the lumbar vertebrae to the sacrum was considered (thoracic vertebrae to the sacrum: odds ratio, 1.21; $p<0.05$; lumbar vertebrae to the sacrum: odds ratio, 2.78; $p<0.01)$. Moreover, low proximal femur BMD in DISH patients raises the fracture risk (odds ratio, $0.47 ; p<0.01$ ).

Conclusions: Many continuous vertebral bone bridges, especially those that extend to the lumbar spine and low proximal femur $\mathrm{BMD}$, are risk factors for fracture in DISH patients.

Keywords: Diffuse idiopathic skeletal hyperostosis; Spinal fractures; Ankylosis

Received Jul 9, 2020; Revised Sep 28, 2020; Accepted Oct 27, 2020

Corresponding author: Mitsuru Furukawa

Department of Orthopedic Surgery, Murayama Medical Center, 2-37-11 Gakuen, Musashimurayamashi, Tokyo 208-0011, Japan Tel: +81-425611221, Fax: +81-425611221, E-mail: furukawa.mitsuru.pa@mail.hosp.go.jp. 


\section{Introduction}

Diffuse idiopathic skeletal hyperostosis (DISH) is a common disorder characterized by irregular calcification. It is the bone formation of soft tissue around the spine joints and the peripheral skeleton or the appendicular skeleton [1]. DISH is different from ankylosing spondylitis (AS) because there is no sacroiliac joint ankylosis and histocompatibility leukocyte antigen-B27 $[2,3]$.

DISH is more common in people aged $>50$ years, with an overall prevalence of $>30 \%$ in people aged $>70$ years $[4,5]$. As per a retrospective study on elderly male patients undergoing computed tomography (CT) of the chest, incomplete osteophytes that are not fused between the vertebrae are transformed into complete bone bridges within an average duration of 5 years. Moreover, DISH progresses with age, and the prevalence of DISH is higher in older subjects [6].

In DISH patients, the bone bridges that connect the intervertebral body create long lever arms that may increase fractures' risk because of minor trauma [7]. There is an increase in the number of spinal injuries with DISH owing to population aging, and bone fracture is a severe problem that commonly leads to permanent spinal cord damage or death $[8,9]$. Research has shown that the diagnosis of spinal fractures is often delayed in DISH patients [10]. Although DISH tends to have bone bridges at the thoracolumbar junction, the number of continuous vertebral bone bridges and the bone bridges' disc levels vary. Thus, it is unclear as to which factors influence the fracture risk [11].

Bone mineral density (BMD) of the lumbar spine and femur proximal extremity is low in AS patients; low BMD is associated with an increased fracture risk $[12,13]$. However, to our knowledge, no study has reported that the BMD of DISH patients is low, and the relationship between low BMD and fracture risk is unknown. Our literature search only showed one study that reported significantly lower BMD in men with both DISH and fractures than that in men with DISH but no fractures, as detected using quantitative computed tomography (QCT) [14]. Thus, to address this research gap, we designed this study to examine how cross-linked bone bridges and BMD influence the fracture risk in DISH patients.

\section{Materials and Methods}

\section{Patients}

We enrolled patients diagnosed with DISH using CT at the Shizuoka City Shimizu Hospital from April 2008 to December 2018. The CT scans (Discovery CT 750HD; GE Healthcare, Chicago, IL, USA) of the thoracic to the sacrum region were performed for 243 patients; 140 were diagnosed with DISH based on a definition of Resnick [15]: confluent ossification of at least four contiguous vertebral bodies, without narrowing of the disc space or sacroiliac ankylosis.

Age, sex, height, weight, glycosylated hemoglobin (HbAlc), calcium level, proximal femur BMD, the maximum number of vertebral bodies that are bony crosslinked with contiguous adjacent vertebrae without interruption (max VB) from the thoracic vertebra to the sacrum, and max VB from the lumbar vertebra to the sacrum were recorded $[16,17]$. The max VB from the thoracic vertebrae to the sacrum was measured from Th1 to the sacrum. The max VB from the lumbar vertebrae to

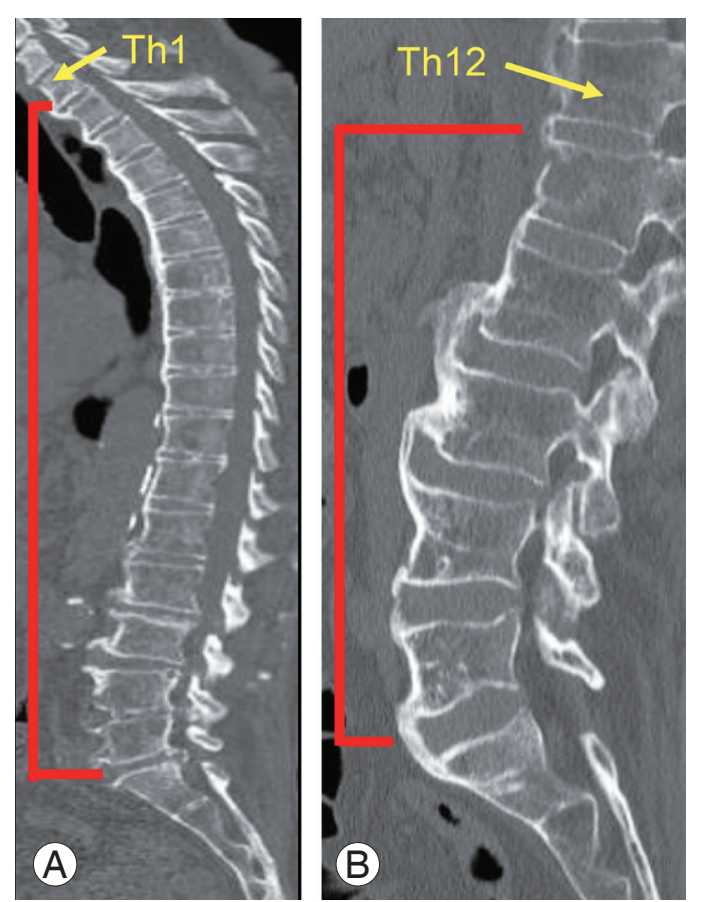

Fig. 1. Max VB was investigated from the thoracic vertebrae to the sacrum, which ranged from Th1 to the sacrum (A) and max VB from the lumbar vertebrae to the sacrum was investigated, which ranged from Th12 to the sacrum (B) in diffuse idiopathic skeletal hyperostosis patients. Max VB, the maximum number of vertebrae that are bony cross-linked with contiguous adjacent vertebrae without interruption. 
the sacrum was measured from Th12 to the sacrum (Fig. 1). Three orthopedic surgeons assessed the max VB using CT. Lumbar and proximal femur BMD was assessed using dual-energy X-ray absorptiometry (DEXA; GE Medical Systems LUNAR, Madison, WI, USA).

The Institutional Review Board of Shizuoka City Shimizu Hospital approved this study, and all the study subjects provided informed consent using the opt-out method on the Shizuoka City Shimizu Hospital website. We conducted this study as per the principles of the Declaration of Helsinki.

\section{Statistical analyses}

The aforementioned items were compared between the fracture group and the non-fracture group using univariate analysis. The mean and standard deviation (SD) values of the fracture and non-fracture groups were calculated and tested using the Mann-Whitney $U$-tests for the above items. With max VB, age, sex, weight, HbAlc, calcium level, and proximal femur BMD as confounding factors, the relationship with these items and the occurrence of vertebral fractures was calculated using logistic regression analysis. Model 1 was not adjusted for the confounding factors, while model 2 was adjusted for the confounding factors. Subsequent investigations were conducted for the subjects in the fracture group. The causes of fracture, fracture level, whether or not the fracture level was included in the range of the max $\mathrm{VB}$, the number of paralysis of the lower limbs, and the number of patients who could not be diagnosed using CT, but could be diagnosed using magnetic resonance imaging (MRI), were included as fracture group parameters. Data are presented as mean and (SD) values. Mann-Whitney $U$-tests were performed to assess the differences between the fracture and nonfracture groups. IBM SPSS Statistics ver. 22.0 (IBM Corp., Armonk, NY, USA) was used for the statistical analyses. Logistic regression analysis was performed using $\mathrm{R}$ software (R Foundation for Statistical Computing, Vienna, Austria). A $p$-value $<0.05$ was considered statistically significant.

\section{Results}

In this study, we assessed 140 patients (98 men and 42 women; average age, 78.6 years); 52 had fractures at the continuous vertebral bodies fused by bone bridges, while
88 did not have fractures. The causes of fracture were indoor fall $(n=36)$, fall from a height $(n=6)$, traffic accident $(n=5)$, fall while walking outdoors $(n=1)$, and unknown cause $(n=4)$. Concerning the fracture level, there were three cases of fracture in the T4 vertebra, one in T5, one in T7, three in T8, four in T9, five in T10, seven in T11, 14 in T12, nine in L1, two in L2, two in L3, one in L4, and one in L5. Five patients had paralysis of the lower limbs. Further, all the fracture levels were included in the max VB range. None of the patients had bone bridges, only from the lumbar vertebrae to the sacrum. Those with lumbar vertebrae bridges had continuous bone bridges from the thoracic vertebrae. Fractures could not be detected using only CT, and short T1 inversion recovery MRI could only be used to diagnose five patients.

In the univariate analysis, the fracture group had a significantly lower proportion of men, weight, calcium level, $\mathrm{HbA1c}$, and proximal femoral BMD and higher age, max VB from the thoracic vertebrae to the sacrum, and max VB from the lumbar vertebra to the sacrum as compared to the non-fracture group (Table 1).

Before adjustment (model 1), the logistic regression analysis associated the higher max VB from the thoracic vertebrae to the sacrum and from the lumbar vertebrae to the sacrum with a higher risk of vertebral fractures (thoracic vertebrae to the sacrum: odds ratio, $1.2 ; p<0.01$; lumbar vertebrae to the sacrum: odds ratio, $1.68 ; p<0.01$ ) (Tables 2, 3).

After adjustment of the confounding factors, logistic regression analysis associated the higher max $\mathrm{VB}$ from the

Table 1. Comparison between patients with and without fracture in diffuse idiopathic skeletal hyperostosis

\begin{tabular}{|c|c|c|}
\hline Variable & $\begin{array}{l}\text { Fracture } \\
(n=52)\end{array}$ & $\begin{array}{c}\text { Non-fracture } \\
\quad(n=88)\end{array}$ \\
\hline Max VB from the thoracic to the sacrum & $11.3 \pm 3.75^{* *}$ & $8.42 \pm 3.62$ \\
\hline Max VB from the lumbar to the sacrum & $3.10 \pm 2.34^{* *}$ & $1.02 \pm 1.51$ \\
\hline Age (yr) & $82.1 \pm 7.82^{*}$ & $77.7 \pm 8.70$ \\
\hline Males & $31(59.6)^{*}$ & $67(76.1)$ \\
\hline Weight (kg) & $54.1 \pm 10.2^{* *}$ & $64.1 \pm 11.1$ \\
\hline Calcium (mg/dL) & $8.68 \pm 0.558^{* *}$ & $9.09 \pm 0.549$ \\
\hline Glycosylated hemoglobin (\%) & $5.83 \pm 0.81^{*}$ & $6.06 \pm 0.69$ \\
\hline Proximal femur bone mineral density $\left(\mathrm{g} / \mathrm{cm}^{2}\right)$ & $0.710 \pm 0.16^{* *}$ & $0.935 \pm 0.22$ \\
\hline
\end{tabular}


Table 2. ORs for the association between some factors and vertebral fracture risk in diffuse idiopathic skeletal hyperostosis patients from the logistic regression models without (model 1) and with (model 2) adjustment for confounding factors

\begin{tabular}{|c|c|c|c|c|}
\hline \multirow{2}{*}{ Variable } & \multicolumn{2}{|c|}{ Model 1} & \multicolumn{2}{|c|}{ Model 2} \\
\hline & OR $(95 \% \mathrm{Cl})$ & $p$-value & OR $(95 \% \mathrm{CI})$ & $p$-value \\
\hline Max VB from thoracic to sacrum & $1.22(1.11-1.35)$ & $<0.001$ & $1.21(1-1.47)$ & $<0.05$ \\
\hline Age (yr) & & & $0.99(0.91-1.08)$ & 0.86 \\
\hline Sex (reference: men) & & & $0.6(0.14-2.56)$ & 0.49 \\
\hline Weights $(\mathrm{kg})$ & & & $0.93(0.86-1.01)$ & 0.08 \\
\hline Calcium (mg/dL) & & & $0.15(0.04-0.61)$ & 0.01 \\
\hline Glycosylated hemoglobin (\%) & & & $1.5(0.64-3.53)$ & 0.35 \\
\hline Proximal femur bone mineral density $\left(0.1 \mathrm{~g} / \mathrm{cm}^{2}\right)$ & & & $0.47(0.3-0.73)$ & $<0.001$ \\
\hline
\end{tabular}

$\mathrm{OR}$, odds ratio; $\mathrm{Cl}$, confidence interval; Max VB, the maximum number of vertebrae that are bony cross-linked with contiguous adjacent vertebrae without interruption.

Table 3. ORs for the association between some factors and vertebral fracture risk in diffuse idiopathic skeletal hyperostosis patients from the logistic regression models without (model 1) and with (model 2) adjustment for confounding factors

\begin{tabular}{|c|c|c|c|c|}
\hline \multirow{2}{*}{ Variable } & \multicolumn{2}{|c|}{ Model 1} & \multicolumn{2}{|c|}{ Model 2} \\
\hline & OR $(95 \% \mathrm{CI})$ & $p$-value & OR $(95 \% \mathrm{CI})$ & $p$-value \\
\hline Max VB from lumbar to sacrum & $1.68(1.38-2.06)$ & $<0.001$ & $2.78(1.64-4.71)$ & $<0.001$ \\
\hline Age (yr) & & & $0.99(0.91-1.08)$ & 0.8 \\
\hline Sex (reference: men) & & & $0.78(0.13-4.89)$ & 0.8 \\
\hline Weight (kg) & & & $0.89(0.81-0.97)$ & 0.01 \\
\hline Calcium (mg/dL) & & & $0.27(0.06-1.20)$ & 0.08 \\
\hline Glycosylated hemoglobin (\%) & & & $2.28(0.76-6.82)$ & 0.14 \\
\hline Proximal femur bone mineral density $\left(0.1 \mathrm{~g} / \mathrm{cm}^{2}\right)$ & & & $0.39(0.22-0.66)$ & 0.01 \\
\hline
\end{tabular}

$\mathrm{OR}$, odds ratio; $\mathrm{Cl}$, confidence interval; $\mathrm{Max} \mathrm{VB}$, the maximum number of vertebrae that are bony cross-linked with contiguous adjacent vertebrae without interruption.

thoracic vertebrae to the sacrum and the lumbar vertebrae to the sacrum with a higher risk of vertebral fractures (thoracic vertebrae to the sacrum: odds ratio, $1.21 ; p<0.05$; lumbar vertebrae to sacrum: odds ratio, $2.78 ; p<0.01$ ) (Tables 2, 3). Moreover, after further adjustment for the confounding factors, low BMD of the proximal femur significantly increased the fracture risk (thoracic vertebrae to the sacrum: odds ratio, $0.47 ; p<0.01$; lumbar vertebrae to sacrum: odds ratio, $0.3 ; p<0.01$ ) (Tables 2,3 ).

\section{Discussion}

This logistic regression analysis included confounding factors that were described as risk factors for fracture. Our findings showed that a high number of continuous vertebral bodies with cross-linked bone bridges in DISH was a significant independent risk factor for fractures. Furthermore, low proximal femur bone density is associated with the risk of fracture in DISH patients.

Fractures with DISH are reverse chance fractures, and many of them fall under type III B hyperextension of the AO classification [18]. The lever arm in DISH comprises a continuous vertebral cross bridging of the vertebrae. Stress concentration in the fracture area can result in fractures. The lever arm is the vertical distance between the axis of rotation and the force's line action. However, a clear definition of a lever arm in DISH is yet to be established. We devised the concept of max VB and proved the association between max VB and fracture risk statistically. We used max VB as an index because the loss of intervertebral discs due to bony cross-linking may increase the fall risk because of poor balance during walking. It may be difficult to adopt a passive approach during a fall. Another reason is that the force applied to the fracture area during a fall in DISH patients may be high. In the present study, we also analyzed the max VB in the following two ways: 
thoracic to sacral and lumbar to sacral. The number of patients with DISH fractures at our hospital was higher for patients whose fractures were cross-linked to the lumbar vertebrae. Although the obvious cause remains unclear, we expect that the lumbar vertebrae have a greater range of motion in the anterior and posterior flexion per vertebrae than the thoracic vertebrae, so cross-linking may be associated with reduced mobility $[19,20]$.

Orthopedic surgeons commonly treat DISH patients, and it is challenging to explain the fracture risk to all DISH patients, especially when this condition is discovered accidentally in a limited consultation time. Therefore, the present results indicate the odds ratio of the max VB from the lumbar vertebrae to the sacrum is higher than that of the max VB from the thoracic vertebrae to the sacrum. It would be more efficient to educate DISH patients in whom lumbar vertebral bone bridges have been found using lumbar radiography about fracture risks. Fractures associated with DISH may progress to dislocation and cause spinal cord injury if a slight dislocation is overlooked during the initial visit. In our study, the fractures in five patients could not be identified on CT and were first identified using MRI. Thus, it is critical to perform CT for all DISH patients who present with severe lumbar back pain after a fall or movement to establish a definitive diagnosis. In the present study, only MRI was performed; however, in centers where MRI is not immediately available, a bone scan may be useful. In any case, we would recommend that all patients undergo an MRI or a bone scan. However, patients who present with dislocation at the first visit may not have DISH. The initial examination is usually a radiography examination of choice, followed by a CT, MRI, or bone scan if DISH is suspected. Therefore, orthopedic surgeons should consider DISH or a DISH-associated fracture in the differential diagnosis based on the initial radiography findings.

In particular, in DISH patients, bone bridges and fractures in the thoracic vertebrae are difficult to determine using radiography because of the overlapping with the lungs and ribs, leading to delayed diagnosis. In contrast, bone bridges in the lumbar spine are easier to identify than those in the thoracic spine. Patients who experience lumbar pain from a fall and those who have bone bridges in their lumbar vertebrae leading from the thoracic vertebrae, but have no fractures identified on lumbar radiography, should undergo CT, MRI, or bone scan of the thoracic to the lumbar spine immediately to confirm the presence of fractures.

As per a previous report, the BMD of the lumbar spine was higher in DISH patients as measured using DEXA; however, there was no significant difference in the BMD using QCT. Another report wherein DEXA was used on cadavers in directions that did not include the bone bridge found no difference in the BMD of the lumbar spine and that of the controls, indicating that the bone bridge leads to an overestimation of the BMD [14,21]. In contrast, two studies reported significantly higher BMD of the femur neck in DISH patients than that in the general population matched for some factors, including age, sex, and weight $[22,23]$.

These reports suggest that the BMD of the femur and spine varies in DISH patients. Lumbar spine BMD measured using the DEXA method shows that bone density is measured at a high level. In contrast, loss of intervertebral mobility causes a localized decrease in the vertebral bone density. Compared with proximal femoral BMD, the two factors are complex and do not give a consistent result for lumbar BMD. In this study, proximal femoral BMD that was unaffected by local factors influencing the fracture risk.

As per the diagnostic criteria for primary osteoporosis, osteoporotic bone loss measured using DEXA was $0.7 \mathrm{~g} /$ $\mathrm{cm}^{2}$ for women in the proximal femur. The cutoff BMD in osteoporosis was $0.67 \mathrm{~g} / \mathrm{cm}^{2}$ for men and $0.61 \mathrm{~g} / \mathrm{cm}^{2}$ for women [24]. Considering the mean BMD in the present study, the values of $0.94 \mathrm{~g} / \mathrm{cm}^{2}$ in the non-fracture group and $0.71 \mathrm{~g} / \mathrm{cm}^{2}$ in the fracture group were above the cutoff, not at all indicative of bone fragility. The lever arm is a major factor that causes fractures without bone fragility in DISH. Moreover, it is noteworthy that the fracture group had lower femoral bone density than the non-fracture group, suggesting that even a small decrease in the BMD can trigger a fracture in DISH patients because of the extreme stress placed on the spine by the lever arm.

This study has certain limitations. This study retrospectively analyzed a small number of patients. The second and third highest numbers of continuous vertebral bone bridges, in addition to max VB, were expected to play complex roles in causing fractures. However, the max VB alone was explained about the fracture risk categorically. Future studies should employ a larger sample size and proactively investigate the fracture risk in the study population. 


\section{Conclusions}

We found that a higher number of continuous vertebrae with cross-linked bone bridges in DISH increased the fracture risk. This finding indicates that DISH patients with continuous vertebral bone bridges up to the lumbar vertebrae need to be educated to avoid falls and examined carefully given their high risk of fractures. Low proximal femur bone density also increases the fracture risk. Even if the bone mass itself is high, a small decrease in bone mass may cause a fracture and requires close monitoring.

\section{Conflict of Interest}

No potential conflict of interest relevant to this article was reported.

\section{Author Contributions}

Mitsuru Furukawa focused on data collection, data analysis, and writing. Kunimasa Okuyama mainly focused on data collection, data analysis. Ken Ninomihya focused on data collection, data analysis, and writing. Yoshiyuki Yato drafted thesis and revised thesis. Masaya Nakamura drafted thesis and revised thesis. Morio Matsumoto drafted thesis and revised thesis.

\section{References}

1. Resnick D, Shaul SR, Robins JM. Diffuse idiopathic skeletal hyperostosis (DISH): Forestier's disease with extraspinal manifestations. Radiology 1975;115:51324.

2. Braun J, Sieper J. Ankylosing spondylitis. Lancet 2007;369:1379-90.

3. Whang PG, Goldberg G, Lawrence JP, et al. The management of spinal injuries in patients with ankylosing spondylitis or diffuse idiopathic skeletal hyperostosis: a comparison of treatment methods and clinical outcomes. J Spinal Disord Tech 2009;22:77-85.

4. Toyoda H, Terai H, Yamada K, et al. Prevalence of diffuse idiopathic skeletal hyperostosis in patients with spinal disorders. Asian Spine J 2017;11:63-70.

5. Julkunen H, Heinonen OP, Knekt P, Maatela J. The epidemiology of hyperostosis of the spine together with its symptoms and related mortality in a general population. Scand J Rheumatol 1975;4:23-7.
6. Kuperus JS, Buckens CF, Sprem J, Oner FC, de Jong PA, Verlaan JJ. The natural course of diffuse idiopathic skeletal hyperostosis in the thoracic spine of adult males. J Rheumatol 2018;45:1116-23.

7. Hendrix RW, Melany M, Miller F, Rogers LF. Fracture of the spine in patients with ankylosis due to diffuse skeletal hyperostosis: clinical and imaging findings. AJR Am J Roentgenol 1994;162:899-904.

8. Young JS, Cheshire JE, Pierce JA, Vivian JM. Cervical ankylosis with acute spinal cord injury. Paraplegia 1977;15:133-46.

9. Caron T, Bransford R, Nguyen Q, Agel J, Chapman J, Bellabarba C. Spine fractures in patients with ankylosing spinal disorders. Spine (Phila Pa 1976) 2010;35:E458-64.

10. Westerveld LA, Verlaan JJ, Oner FC. Spinal fractures in patients with ankylosing spinal disorders: a systematic review of the literature on treatment, neurological status and complications. Eur Spine J 2009;18:145-56.

11. Nishimura S, Nagoshi N, Iwanami A, et al. Prevalence and distribution of diffuse idiopathic skeletal hyperostosis on whole-spine computed tomography in patients with cervical ossification of the posterior longitudinal ligament: a multicenter study. Clin Spine Surg 2018;31:E460-5.

12. Karberg K, Zochling J, Sieper J, Felsenberg D, Braun J. Bone loss is detected more frequently in patients with ankylosing spondylitis with syndesmophytes. J Rheumatol 2005;32:1290-8.

13. Singh HJ, Nimarpreet K, Ashima, Das S, Kumar A, Prakash S. Study of bone mineral density in patients with ankylosing spondylitis. J Clin Diagn Res 2013;7:2832-5.

14. Diederichs G, Engelken F, Marshall LM, et al. Diffuse idiopathic skeletal hyperostosis (DISH): relation to vertebral fractures and bone density. Osteoporos Int 2011;22:1789-97.

15. Resnick D. Diffuse idiopathic skeletal hyperostosis (DISH). West J Med 1976;124:406-7.

16. De Laet C, Kanis JA, Oden A, et al. Body mass index as a predictor of fracture risk: a meta-analysis. Osteoporos Int 2005;16:1330-8.

17. Johansson H, Kanis JA, Oden A, et al. A meta-analysis of the association of fracture risk and body mass index in women. J Bone Miner Res 2014;29:223-33.

18. Tan T, Huang MS, Hunn MK, Tee J. Patients with an- 
kylosing spondylitis suffering from AO type B3 traumatic thoracolumbar fractures are associated with increased frailty and morbidity when compared with patients with diffuse idiopathic skeletal hyperostosis. J Spine Surg 2019;5:425-32.

19. Morita D, Yukawa Y, Nakashima H, et al. Range of motion of thoracic spine in sagittal plane. Eur Spine J 2014;23:673-8.

20. Yukawa Y, Matsumoto T, Kollor H, et al. Local sagittal alignment of the lumbar spine and range of motion in 627 asymptomatic subjects: age-related changes and sex-based differences. Asian Spine J 2019;13:663-71.

21. Westerveld LA, Verlaan JJ, Lam MG, et al. The influence of diffuse idiopathic skeletal hyperostosis on bone mineral density measurements of the spine. Rheumatology (Oxford) 2009;48:1133-6.
22. Sohn S, Chung CK, Han I, Park SB, Kim H. Increased bone mineral density in cervical or thoracic diffuse idiopathic skeletal hyperostosis (DISH): a case-control study. J Clin Densitom 2018;21:68-74.

23. Furukawa M, Okuyama K, Kawano Y, et al. Femur bone mineral density and pentosidine level distinguish ankylosing spinal disorder patients with and without sacroiliac ankylosis. Spine Surg Relat Res 2020;4:333-40.

24. Soen S, Fukunaga H, Sugimoto T, et al. New diagnostic criteria for primary osteoporosis (2012 revision), diagnostic criteria for primary osteoporosis (2012 revision). Osteoporos Jpn [Internet]. 2013 [cited 2020 Jun 20];21:12-4. Available from: http://jsbmr.umin. jp/guide/pdf/g-guideline.pdf. 